\title{
Phenomenological aspects of evolution in transverse momentum dependent parton distribution and fragmentation functions
}

\section{Miguel G. Echevarría}

NIKHEF and Department of Physics and Astronomy, VU University Amsterdam, De Boelelaan 1081, NL-1081 HV Amsterdam, the Netherlands

E-mail: m.q.echevarriaenikhef.n]

\section{Ahmad Idilbi}

Department of Physics, Pennsylvania State University, Uni versity Park, PA 16802, USA

E-mail: auil3epsu.edu

\section{Ignazio Scimemi*}

Departamento de Física Teórica II, Universidad Complutense de Madrid (UCM), 28040

Madrid, Spain

E-mail: ionaziosdfis.ucm.es

We review some of the recent progress in the studies of properties of transverse momentum dependent parton distribution and fragmentation functions with or without specified polarization. In particular we argue that the evolution for all these quantities is universal. This result relies on established, leading twist factorization theorems for the relevant underlying processes. Using the current knowledge of the perturbative contributions we can resum large logarithms up to next-to-next-to-leading logarithmic accuracy.

XXII International Worskhop on Deep-Inelastic Scattering and Related Subjects April 28 - May 2, 2014

Warsaw, Poland

\footnotetext{
${ }^{*}$ Speaker.
} 
Our understanding of transverse momentum dependent observables has strongly improved recently with the achievement of the proper definition for the relevant hadronic quantities involved, i.e. the transverse momentum dependent functions (TMDs) [W, 口, []]. These appear both in the initial state, as TMD parton distribution functions (TMDPDFs), and in the final state, as TMD fragmentation functions (TMDFFs). In the $q_{T}$-spectrum of semi-inclusive deep-inelastis scattering (SIDIS) or Drell-Yan (DY) processes, different TMDs contribute, at leading-twist, to the factorization of the QCD hadronic tensor depending on the polarization of the involved hadrons/partons. In order to study the hadronic spin structure one needs to properly define all these functions, which at leading twist there are sixteen of them, eight TMDPDFs and eight TMDFFs. Their evolution turns out to be driven by a universal evolution kernel, which allows us to resum the large logarithms at next-to-next-to-leading-logarithmic (NNLL) accuracy, given the current knowledge of the relevant perturbative ingredients. The universal evolution kernel is a key ingredient in the interpretation of experimental data and the phenomenological extraction of TMDs.

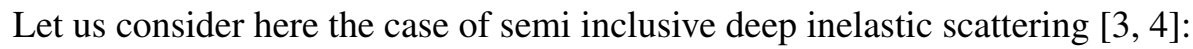

$$
l(k)+N(P) \rightarrow l^{\prime}\left(k^{\prime}\right)+h\left(P_{h}\right)+X\left(P_{X}\right),
$$

where $l\left(l^{\prime}\right)$ is the incoming (outgoing) lepton, $N$ is the nucleon and $h$ is the detected hadron, for which we measure its transverse momentum. This process is commonly described in terms of the following Lorentz invariants,

$$
x_{B}=\frac{Q^{2}}{2 P \cdot q}, \quad y=\frac{P \cdot q}{P \cdot l}, \quad z_{h}=\frac{P \cdot P_{h}}{P \cdot q} .
$$

The photon carries momentum $q=k-k^{\prime}$ with $q^{2}=-Q^{2}$. In the Breit frame, the incoming nucleon $N$ is traveling along the $+z$-direction, with $n$-collinear momentum $P$, and the photon is $\bar{n}$-collinear, traveling along the $-z$-direction ${ }^{1}$. The outgoing hadron $h$ has a momentum $P_{h}$ mainly along the $-z$-direction, acquiring a transverse momentum $P_{h \perp}$. The axial four-spin vectors of the nucleon and the hadron, $S$ and $S_{h}$ respectively, satisfy $S^{2}=S_{h}^{2}=-1$ and $S \cdot P=S_{h} \cdot P_{h}=0$. The differential cross section for SIDIS under one photon exchange can then be written as

$$
\frac{d^{5} \sigma}{d x_{B} d y d z_{h} d^{2} P_{h \perp}}=\frac{\pi \alpha_{e m}^{2}}{2 Q^{4}} y L_{\mu v} W^{\mu \nu}
$$

The leptonic tensor $L_{\mu v}$ is $L_{\mu v}=2\left(k_{\mu} k_{v}^{\prime}+k_{v} k_{\mu}^{\prime}-g_{\mu v} k \cdot k^{\prime}\right)+2 i \lambda_{l} \varepsilon_{\mu v \rho \sigma} l^{\rho} q^{\sigma}$, where we have summed over the spin of the final lepton, $s_{l^{\prime}}$. The hadronic tensor $W^{\mu \nu}$ is given by

$$
\begin{aligned}
W^{\mu v} & =\frac{1}{(2 \pi)^{4}} \frac{1}{z} \sum_{X} \int \frac{d^{3} P_{X}}{(2 \pi)^{3} 2 E_{X}}(2 \pi)^{4} \delta^{(4)}\left(P+q-P_{h}-P_{X}\right)\left\langle P S\left|J^{\mu \dagger}(0)\right| X ; P_{h} S_{h}\right\rangle\left\langle X ; P_{h} S_{h}\left|J^{v}(0)\right| P S\right\rangle \\
& =\frac{1}{z} \sum_{X} \int \frac{d^{4} r}{(2 \pi)^{4}} e^{i q \cdot r}\left\langle P S\left|J^{\mu \dagger}(r)\right| X ; P_{h} S_{h}\right\rangle\left\langle X ; P_{h} S_{h}\left|J^{v}(0)\right| P S\right\rangle
\end{aligned}
$$

where the sum over the undetected hadrons in the final state, $X$, includes as well the integration over $P_{X}$. The factorization of the cross section can be stated only when the photon energy probe is

\footnotetext{
${ }^{1} \mathrm{~A}$ generic vector $v^{\mu}$ is decomposed as $v^{\mu}=\bar{n} \cdot v \frac{n^{\mu}}{2}+n \cdot v \frac{\bar{n}^{\mu}}{2}+v_{\perp}^{\mu}=\left(\bar{n} \cdot v, n \cdot v, v_{\perp}^{\mu}\right)=\left(v^{+}, v^{-}, v_{\perp}^{\mu}\right)$, with $n, \bar{n}$ such that $n^{2}=\bar{n}^{2}=0$ and $n \cdot \bar{n}=2$ and $v_{T}$ othogonal to $n, \bar{n}$ (the usual choice being $n=(1,0,0,1), \bar{n}=(1,0,0,-1)$ ), We also use $v_{T}=\left|v_{\perp}\right|$, so that $v_{\perp}^{2}=-v_{T}^{2}$.
} 
much bigger than the hadronization scale $\left(\Lambda_{Q C D} \sim 1 \mathrm{GeV}\right)$ and/or the observed transverse momenta $Q^{2} \gg \max \left(\Lambda_{Q C D}, q_{T}\right)$. The first step of factorization of the SIDIS hadronic tensor is done by matching the full QCD current ${ }^{2}$ onto the effective theory (SCET) counterpart

$$
J_{\mathrm{QCD}}^{\mu}=\sum_{q} e_{q} \bar{\psi} \gamma^{\mu} \psi \longrightarrow J_{\mathrm{SCET}}^{\mu}=C\left(Q^{2} / \mu^{2}\right) \sum_{q} e_{q} \bar{\xi}_{\bar{n}} \tilde{W}_{\bar{n}}^{T} \tilde{S}_{\bar{n}}^{T \dagger} \gamma^{\mu} S_{n}^{T} \tilde{W}_{n}^{T \dagger} \xi_{n}
$$

which contains soft and collinear modes. The Wilson coefficient $C\left(Q^{2} / \mu^{2}\right)$ can be extracted from the finite terms of the calculation of the (full QCD) quark form factor in pure dimensional regularization, and it is known up to $\mathscr{O}\left(\alpha_{s}^{2}\right)$ [可. One of the key ingredients of the SCET machinery is the decoupling of the Hilbert space of the partonic states into three subspaces corresponding to $n$-collinear, $\bar{n}$-collinear and soft modes. After this decoupling, standard manipulations lead to the following form of the hadronic tensor

$$
W^{\mu v}=H\left(Q^{2} / \mu^{2}\right) \frac{1}{N_{c}} \sum_{q} e_{q} \int \frac{d^{4} r}{(2 \pi)^{4}} e^{i q \cdot r} \operatorname{Tr}\left[\Phi^{(0)}(r ; P, S) \gamma^{\mu} \Delta^{(0)}\left(r ; P_{h}, S_{h}\right) \gamma^{v}\right] S(r)+\mathscr{O}\left(\frac{q_{T}}{Q}\right),
$$

where $H\left(Q^{2} / \mu^{2}\right)=\left|C\left(Q^{2} / \mu^{2}\right)\right|^{2}$ and

$$
\begin{aligned}
\Phi_{i j}^{(0)}(r ; P, S) & =\left.\left\langle P S\left|\left[\bar{\xi}_{n j} \tilde{W}_{n}^{T}\right](r)\left[\tilde{W}_{n}^{T^{\dagger}} \xi_{n i}\right](0)\right| P S\right\rangle\right|_{\mathrm{zb} \text { subtracted }}, \\
\Delta_{i j}^{(0)}\left(r ; P_{h}, S_{h}\right) & =\left.\frac{1}{z} \sum_{\chi}\left\langle 0\left|\left[\tilde{W}_{\bar{n}}^{T^{\dagger}} \xi_{\bar{n} i}\right](r)\right| X ; P_{h} S_{h}\right\rangle\left\langle X ; P_{h} S_{h}\left|\left[\bar{\xi}_{\bar{n}} \tilde{W}_{\bar{n} j}^{T}\right](0)\right| 0\right\rangle\right|_{\text {zb subtracted }}, \\
S(r) & =\frac{1}{N_{c}}\left\langle 0\left|\operatorname{Tr}\left[S_{n}^{T \dagger} \tilde{S}_{\bar{n}}^{T}\right](r)\left[\tilde{S}_{\bar{n}}^{T \dagger} S_{n}^{T}\right](0)\right| 0\right\rangle .
\end{aligned}
$$

The "zb-subtracted" stands for zero-bin subtraction which means that one needs to subtract the soft momentum modes contributions from the naively calculated collinear matrix elements, thereby obtaining the so-called "pure collinear" matrix elements.

Since the incoming and outgoing quarks are $n$-collinear and $\bar{n}$-collinear, respectively, the virtual photon momentum is hard, $q=k_{\bar{n}}-k_{n} \sim Q(1,1, \lambda)$, and thus in the exponential in Eq. (G) we have $r \sim(1 / Q)(1,1,1 / \lambda)$. Then, we need to Taylor expand the previous result and consider only the leading order contributions in $\lambda$. Thus we get

$$
\begin{aligned}
W^{\mu v} & =H\left(Q^{2} / \mu^{2}\right) \frac{2}{N_{c}} \sum_{q} e_{q} \int d^{2} k_{n \perp} d^{2} k_{\bar{n} \perp} d^{2} k_{s \perp} \delta^{(2)}\left(q_{\perp}+k_{n \perp}-k_{\bar{n} \perp}+k_{s \perp}\right) \\
& \times \operatorname{Tr}\left[\Phi^{(0)}\left(x, k_{n \perp}, S\right) \gamma^{\mu} \Delta^{(0)}\left(z, k_{\bar{n} \perp}, S_{h}\right) \gamma^{v}\right] S\left(k_{s \perp}\right)
\end{aligned}
$$

where

$$
\begin{aligned}
\Phi_{i j}^{(0)}\left(x, k_{n \perp}, S\right) & =\left.\frac{1}{2} \int \frac{d y^{-} d^{2} y_{\perp}}{(2 \pi)^{3}} e^{-i\left(\frac{1}{2} y^{-} k_{n}^{+}-y_{\perp} \cdot k_{n \perp}\right)}\left\langle P S\left|\left[\bar{\xi}_{n j} \tilde{W}_{n}^{T}\right]\left(0^{+}, y^{-}, y_{\perp}\right)\left[\tilde{W}_{n}^{T \dagger} \xi_{n i}\right](0)\right| P S\right\rangle\right|_{\text {zb subtracted }}, \\
\Delta_{i j}^{(0)}\left(z, \hat{P}_{h \perp}, S_{h}\right) & =\frac{1}{2} \int \frac{d y^{+} d^{2} y_{\perp}}{(2 \pi)^{3}} e^{i\left(\frac{1}{2} y^{+} k_{\bar{n}}^{-}-y_{\perp} \cdot k_{\bar{n} \perp}\right)} \\
& \times\left.\frac{1}{z} \sum_{\chi}\left\langle 0\left|\left[\tilde{W}_{\bar{n}}^{T \dagger} \xi_{\bar{n} i}\right]\left(y^{+}, 0^{-}, y_{\perp}\right)\right| X ; P_{h} S_{h}\right\rangle\left\langle X ; P_{h} S_{h}\left|\left[\bar{\xi}_{\bar{n}} \tilde{W}_{\bar{n} j}^{T}\right](0)\right| 0\right\rangle\right|_{\text {zb subtracted }} \\
S\left(k_{S \perp}\right) & =\int \frac{d^{2} y_{\perp}}{(2 \pi)^{2}} e^{i y_{\perp} \cdot k_{s \perp}} \frac{1}{N_{c}}\left\langle 0\left|\operatorname{Tr}\left[S_{n}^{T \dagger} \tilde{S}_{\bar{n}}^{T}\right]\left(0^{+}, 0^{-}, y_{\perp}\right)\left[\tilde{S}_{\bar{n}}^{T \dagger} S_{n}^{T}\right](0)\right| 0\right\rangle
\end{aligned}
$$

\footnotetext{
${ }^{2}$ We consider the case of a one photon exchange. The extension to $W$ and $Z$ bosons exchange is straightforward.
} 
For the $\Phi$ correlator we have $k_{n}^{+}=x P^{+}$, while for the $\Delta$ correlator we have $k_{\bar{n}}^{-}=\hat{P}_{h}^{-} / z$ and $k_{n \perp}=$ $-\hat{P}_{h \perp} / z . \hat{P}_{h \perp}$ can be interpreted as the transverse momentum of the outgoing hadron $h$ in a frame where the fragmenting quark has no transverse momentum.

When calculated perturbatively (i.e. partonically) the three matrix elements above contain, individually, rapidity divergences and hence neither one of them is well-defined. In order to properly define the TMDPDFs and TMDFFs, the soft function is split into two pieces in rapidity space, as was shown in Ref. [D],

$$
\begin{gathered}
\tilde{S}\left(b_{T} ; \frac{Q^{2} \mu^{2}}{\Delta^{+} \Delta^{-}}, \mu^{2}\right)=\tilde{S}_{-}\left(b_{T} ; \zeta_{F}, \mu^{2} ; \Delta^{-}\right) \tilde{S}_{+}\left(b_{T} ; \zeta_{D}, \mu^{2} ; \Delta^{+}\right), \\
\tilde{S}_{-}\left(b_{T} ; \zeta_{F}, \mu^{2} ; \Delta^{-}\right)=\sqrt{\tilde{S}\left(\frac{\Delta^{-}}{p^{+}}, \alpha \frac{\Delta^{-}}{\bar{p}^{-}}\right)}, \quad \tilde{S}_{+}\left(b_{T} ; \zeta_{D}, \mu^{2} ; \Delta^{+}\right)=\sqrt{\tilde{S}\left(\frac{1}{\alpha} \frac{\Delta^{+}}{p^{+}}, \frac{\Delta^{+}}{\bar{p}^{-}}\right)},
\end{gathered}
$$

where in the soft functions under the square roots we have explicitly specified the dependence on the $\Delta$-regulator parameters that regulate the soft Wilson lines in the $n$ - and $\bar{n}$-directions. $\zeta_{F}$ and $\zeta_{D}$ are fractions of $Q^{2}$ satisfying $\zeta_{F} \zeta_{D}=Q^{4}$, where $\zeta_{F}=Q^{2} / \alpha$ and $\zeta_{D}=\alpha Q^{2}$ with $\alpha$ an arbitrary boost-invariant real number (one can call it the "soft splitting number"). $p^{+}$and $\bar{p}^{-}$stand for the two large collinear momentum components carried by the incoming and outgoing partons, respectively, that initiate the DIS hard reaction. The superscript $\sim$ refers to quantities calculated in impact parameter space (IPS). We emphasize the fact that, as was mentioned in Ref. [R], the splitting of the soft function in rapidity space does not depend on a particular regulator.

In order to properly define the TMDs, the two pieces of the soft function presented above are combined with the two quark correlators $(\Phi$ and $\Delta)$. The resulting quantities are free from rapidity divergences and hence can be considered as a valid hadronic quantities. Thus, the TMDPDFs are defined by

$$
F_{i j}\left(x, k_{n \perp}, S ; \zeta_{F}, \mu^{2} ; \Delta^{-}\right)=\int d^{2} b_{\perp} e^{i b_{\perp} \cdot k_{n \perp}} \tilde{\Phi}_{i j}^{(0)}\left(x, b_{\perp}, S ; \mu^{2} ; \Delta^{-}\right) \tilde{S}_{-}\left(b_{T} ; \zeta_{F}, \mu^{2} ; \Delta^{-}\right),
$$

while for the TMDFFs we have

$$
D_{i j}\left(z, \hat{P}_{h \perp}, S_{h} ; \zeta_{D}, \mu^{2} ; \Delta^{+}\right)=\int d^{2} b_{\perp} e^{-i b_{\perp} \cdot k_{\bar{n} \perp}} \tilde{\Delta}_{i j}^{(0)}\left(z, b_{\perp}, S_{h} ; \mu^{2} ; \Delta^{+}\right) \tilde{S}_{+}\left(b_{T} ; \zeta_{D}, \mu^{2} ; \Delta^{+}\right) .
$$

With the definitions above we can write the hadronic tensor as

$$
\begin{aligned}
W^{\mu v} & =H\left(Q^{2} / \mu^{2}\right) \frac{2}{N_{c}} \sum_{q} e_{q} \int d^{2} k_{n \perp} d^{2} k_{\bar{n} \perp} \delta^{(2)}\left(q_{\perp}+k_{n \perp}-k_{\bar{n} \perp}\right) \\
& \times \operatorname{Tr}\left[F\left(x, k_{n \perp}, S ; \zeta_{F}, \mu^{2}\right) \gamma^{\mu} D\left(z, \hat{P}_{h \perp}, S_{h} ; \zeta_{D}, \mu^{2}\right) \gamma^{v}\right]
\end{aligned}
$$

The evolution of the different TMDs is governed through their anomalous dimensions which are defined as follows

$$
\begin{aligned}
& \frac{d}{d \ln \mu} \ln \tilde{F}_{f / N}\left(x, z b_{\perp}, S ; \zeta_{F}, \mu^{2}\right) \equiv \gamma_{F}\left(\alpha_{s}(\mu), \ln \frac{\zeta_{F}}{\mu^{2}}\right), \\
& \frac{d}{d \ln \mu} \ln \tilde{D}_{h / f}\left(z, b_{\perp}, S_{h} ; \zeta_{D}, \mu^{2}\right) \equiv \gamma_{D}\left(\alpha_{s}(\mu), \ln \frac{\zeta_{D}}{\mu^{2}}\right) .
\end{aligned}
$$


Notice that the evolution of the TMDs with respect to the factorization scale $\mu$ is related to that of the hard part, $H$. Since the hadronic tensor does not depend on the factorization scale, the anomalous dimensions $\gamma_{F}$ and $\gamma_{D}$ and the one of the hard part, $\gamma_{H}$ are connected by

$$
\gamma_{H}=\frac{d}{d \ln \mu} H\left(Q^{2} / \mu^{2}\right)=-\gamma_{F}\left(\alpha_{s}(\mu), \ln \frac{\zeta_{F}}{\mu^{2}}\right)-\gamma_{D}\left(\alpha_{s}(\mu), \ln \frac{\zeta_{D}}{\mu^{2}}\right)
$$

and thus

$$
\begin{aligned}
& \gamma_{F}\left(\alpha_{s}(\mu), \ln \frac{\zeta_{F}}{\mu^{2}}\right)=-\Gamma_{\text {cusp }}\left(\alpha_{s}(\mu)\right) \ln \frac{\zeta_{F}}{\mu^{2}}-\gamma^{V}\left(\alpha_{s}(\mu)\right), \\
& \gamma_{D}\left(\alpha_{s}(\mu), \ln \frac{\zeta_{D}}{\mu^{2}}\right)=-\Gamma_{\text {cusp }}\left(\alpha_{s}(\mu)\right) \ln \frac{\zeta_{D}}{\mu^{2}}-\gamma^{V}\left(\alpha_{s}(\mu)\right) .
\end{aligned}
$$

It should be mentioned that the splitting of $\gamma_{H}$ into $\gamma_{F}$ and $\gamma_{D}$ given in the last equation is unique following the restriction of $\zeta_{F} \zeta_{D}=Q^{4}$. The coefficients of the perturbative expansions of $\Gamma_{\text {cusp }}$ and $\gamma^{V}$ are known up to three loops and they are collected in [G].

On the other hand, the TMDs depend as well on $Q^{2}$ through the variables $\zeta_{F}$ and $\zeta_{D}$. This can be easily verified, e.g., by considering the NLO results for the unpolarized TMDPDF (see Eq. (21) in Ref. [[]]) or for the unpolarized TMDFF. We next discuss the evolution of all TMDs with respect to $Q^{2}$, or equivalently $\zeta_{F}$ and $\zeta_{D}$.

The starting point is Eqs. (ㅁ)-(ㅁ) . In IPS where the convolution becomes a simple product, one has the following:

$$
\begin{aligned}
\ln F_{i j}\left(x, b_{\perp}, S ; \zeta_{F}, \mu^{2} ; \Delta^{-}\right) & =\ln \tilde{\Phi}_{i j}^{(0)}\left(x, b_{\perp}, S ; \mu^{2} ; \Delta^{-}\right)+\ln \tilde{S}_{-}\left(b_{T} ; \zeta_{F}, \mu^{2} ; \Delta^{-}\right) \\
\ln D_{i j}\left(z, b_{\perp}, S_{h} ; \zeta_{D}, \mu^{2} ; \Delta^{+}\right) & =\ln \tilde{\Delta}_{i j}^{(0)}\left(z, b_{\perp}, S_{h} ; \mu^{2} ; \Delta^{+}\right)+\ln \tilde{S}_{+}\left(b_{T} ; \zeta_{D}, \mu^{2} ; \Delta^{+}\right) .
\end{aligned}
$$

We notice that the $\zeta$-dependence in Eqs. (ID7) lies completely in the soft factors, while the pure collinear contributions $\left(\tilde{\Phi}^{(0)}\right.$ and $\left.\tilde{\Delta}^{(0)}\right)$ are free from any $\zeta$-dependence. This observation is important. Each pure collinear contribution depends solely on one collinear sector: $n$-collinear for the TMDPDFs and $\bar{n}$-collinear for the TMDFFs ${ }^{3}$. As such, it is impossible to generate any $Q^{2}$ dependence in those quantities since the only way that the $Q^{2}$ can appear (either in the collinear or the soft factors) is through the (boost invariant) combination of $p^{+} \bar{p}^{-}=Q^{2}$ (here we are assuming that we are in the Breit frame). On the other hand the soft gluon radiation has no preferred collinear direction (both light-cone momentum components have the same scaling) and the soft factors do include $Q^{2}$-dependence through a term of the form $\log \left(\Delta^{+} \Delta^{-} / Q^{2} \mu^{2}\right)$ (see Eq. (18) in Ref. [D]). Moreover, in Ref. []], where we considered the DY kinematics, it was shown that to all orders in perturbation theory, $\ln \tilde{S}$ has a single logarithmic dependence on $\ln \left(\Delta^{+} \Delta^{-} / Q^{2} \mu^{2}\right)$, so that one can write

$$
\ln \tilde{S}_{ \pm}=\frac{1}{2} \mathscr{R}_{s}\left(b_{T}, \alpha_{s}\right)+D\left(b_{T}, \alpha_{s}\right) \ln \left(\frac{\left(\Delta^{ \pm}\right)^{2}}{\zeta_{F} \mu^{2}}\right)
$$

\footnotetext{
${ }^{3}$ This is not the case for the naive collinear contributions, since such quantities involve soft contamination in each of them. This soft contamination "connects" the two collinear sectors and thus a non-valid $Q^{2}$-dependence appears in the collinear contributions to both the TMDPDFs and the TMDFFs. Thus avoiding double counting is crucial.
} 
where, as already mentioned before, $\zeta_{F}=Q^{2} / \alpha$ and $\zeta_{D}=\alpha Q^{2}$ with $\alpha$ the arbitrary boost-invariant soft splitting number.

The soft function results to be Hermitian and contains a single logarithm of $Q^{2}$ to all orders in perturbation theory. Then, when going from time-like kinematics (DY) to space-like ones (DIS), it turns out to be universal. Thus the arguments of Ref. [0] for the splitting of the soft function carry over straightforwardly to SIDIS kinematics. Combining this observation with Eqs. (ㅍ), we get that the $Q^{2}$-dependence of the TMDPDFs and TMDFFs is governed by the same function:

$$
\frac{d}{d \ln \zeta_{F}} \ln \tilde{F}_{f / N}\left(x, z b_{\perp}, S ; \zeta_{F}, \mu^{2}\right)=-D\left(b_{T} ; \mu^{2}\right) ; \quad \frac{d}{d \ln \zeta_{D}} \ln \tilde{D}_{h / f}\left(z, b_{\perp}, S_{h} ; \zeta_{D}, \mu^{2}\right)=-D\left(b_{T} ; \mu^{2}\right)
$$

Then given Eqs. (표담) and the $\mu$-independence of the hadronic tensor we have at all orders

$$
\frac{d D}{d \ln \mu}=\Gamma_{\text {cusp }}
$$

The universal $D$ function has a valid perturbative expansion only for low values of $b_{\perp}$. For large $b_{\perp}$ one needs to parameterize it and extract it from experiment, although current data do not seem to be precise enough for this purpose, as it is shown in Ref. [D]. We notice that the $D$-function and the anomalous dimension are not the only universal pieces of the TMD as noted in Ref. [ $[$ ] (we refer to the Q-independent terms called $h_{\Gamma, \gamma}$ in that reference). In that work the universal properties of TMDs derived in Ref. [四] are exploited in order to constrain their non-perturbative inputs, obtaining a very precise determination.

\section{Acknowledgments}

This work is supported by the Spanish MEC, FPA2011-27853-CO2-02. M.G.E. is supported by the "Stichting voor Fundamenteel Onderzoek der Materie" (FOM), which is financially supported by the "Nederlandse Organisatie voor Wetenschappelijk Onderzoek" (NWO).

\section{References}

[1] M. G. Echevarria, A. Idilbi and I. Scimemi, JHEP 1207 (2012) 002 [arXiv:1111.4996 [hep-ph]].

[2] M. G. Echevarria, A. Idilbi and I. Scimemi, Phys. Lett. B 726 (2013) 795 [arXiv:1211.1947 [hep-ph]].

[3] J. Collins, "Foundations of perturbative QCD," (Cambridge monographs on particle physics, nuclear physics and cosmology. 32)

[4] M. G. Echevarria, A. Idilbi and I. Scimemi, Phys. Rev. D 90 (2014) 014003 [arXiv:1402.0869 [hep-ph]].

[5] A. Idilbi, X. -d. Ji and F. Yuan, Nucl. Phys. B 753 (2006) 42 [hep-ph/0605068].

[6] M. G. Echevarria, A. Idilbi, A. Schäfer and I. Scimemi, Eur. Phys. J. C 73 (2013) 2636 [arXiv:1208.1281 [hep-ph]].

[7] U. D’Alesio, M. G. Echevarria, S. Melis and I. Scimemi, arXiv:1407.3311 [hep-ph]. 